This item was submitted to Loughborough's Research Repository by the author.

Items in Figshare are protected by copyright, with all rights reserved, unless otherwise indicated.

\title{
Non-parametric statistical learning for URLLC transmission rate control
}

PLEASE CITE THE PUBLISHED VERSION

https://doi.org/10.1109/ICC42927.2021.9500926

PUBLISHER

IEEE

VERSION

AM (Accepted Manuscript)

\section{PUBLISHER STATEMENT}

(c) 2021 IEEE. Personal use of this material is permitted. Permission from IEEE must be obtained for all other uses, in any current or future media, including reprinting/republishing this material for advertising or promotional purposes, creating new collective works, for resale or redistribution to servers or lists, or reuse of any copyrighted component of this work in other works.

\section{LICENCE}

All Rights Reserved

\section{REPOSITORY RECORD}

Zhang, Wenheng, Mahsa Derakhshani, and Sangarapillai Lambotharan. 2021. "Non-parametric Statistical Learning for URLLC Transmission Rate Control". Loughborough University. https://hdl.handle.net/2134/14096053.v1. 


\title{
Non-parametric Statistical Learning for URLLC Transmission Rate Control
}

\author{
Wenheng Zhang*, Mahsa Derakhshani*, Sangarapillai Lambotharan* \\ *Wolfson School of Mechanical, Electrical and Manufacturing Engineering, Loughborough University, UK \\ Emails: W.Zhang2@lboro.ac.uk, M.Derakhshani@lboro.ac.uk, S.Lambotharan@lboro.ac.uk
}

\begin{abstract}
As an important service for 5G communications, ultra-reliable low-latency communications (URLLC) support emerging mission-critical applications, such as factory automation and autonomous driving. For such applications, the probability of failing to successfully transmit URLLC packets should be below a certain threshold. However, in the case of limited knowledge of the channel distribution, achieving such a reliability target requires precise channel modeling. In this paper, we study applying a non-parametric statistical learning approach (i.e. kernel density estimation (KDE)) to estimate the information of the wireless transmission environment (i.e. the probability density function of the channel distribution). Based on the estimated cumulative distribution function, a transmission rate control technique has been developed and the corresponding reliability has been investigated using two measures representing the average performance and the confidence level. Moreover, this paper compares the performance of $\mathrm{KDE}$ and traditional empirical estimation scheme. The results show that KDE achieves a high level of confidence in guaranteeing the reliability constraint despite of the limited number of training data when choosing a suitable kernel bandwidth.
\end{abstract}

Index Terms-Statistical learning, non-parametric models, kernel density estimation, URLLC, channel uncertainty.

\section{INTRODUCTION}

As one of the key use cases of $5 \mathrm{G}$ technologies, URLLC has the potential to support extensive applications that rely on mission-critical links such as industrial automation [1]-[3]. 3GPP states that in such applications, the system should serve each packet within a deadline (i.e., one millisecond) and the successful service probability of channel transmissions should be guaranteed no less than a target value (i.e., packet error rate is less than $10^{-5}$, or even down to $10^{-9}$ ) [4]. Due to stringent requirements of URLLC, the principles of resource allocation for this use case are distinct from enhanced mobile broadband (eMBB) and thus new schemes have been investigated (e.g., [5], [6]) to schedule URLLC packets and/or optimize the blocklength and power allocation when channel information is available.

A meaningful URLLC transmission should rely on the statistical channel model under which its reliability can be expressed in terms of an average service outage probability. However, the channel state information in a majority of potential transmission systems are uncertain which would affect the rigid requirements of URLLC. Therefore, learning channel

This work was supported by the Royal Academy of Engineering under the Leverhulme Trust Research Fellowship scheme (DerakhshaniLTRF1920 \16\67). statistics is paramount for URLLC that is highly susceptible to rare events such as severe fading [7]. In [8], it has been shown that a model mismatch in channel estimation has a severe impact on the realized reliability in URLLC.

Currently, the foregone technologies still need to be improved in terms of the perspective of data analysis to realize an efficient transmission of URLLC. Learning frameworks need to be devised to capture channel statistics and model the performance of various transmission channels in the regime of ultra-reliable transmission. For instance, the authors in [9] put forward a novel algorithm which was implemented on top of extreme value theory for statistical modeling of the URLLC rare events. Furthermore, in [8], statistical learning algorithms were proposed for URLLC systems to estimate the channel distributions under parametric and non-parametric cases. It has been deduced that the approach based on parametric channel model is sensitive to model mismatches and hence not desirable when the accurate knowledge of channel distribution models is not available. Thus, a promising direction is to focus on the non-parametric statistical learning for the URLLC channel estimation. However, the proposed empirical nonparametric approach requires a prohibitively large number of training samples to meet the required targets.

In this paper, for URLLC systems, we propose a statistical learning algorithm applying kernel density estimation (KDE) to estimate the probability density function (PDF) of channel distribution and further maintain the URLLC reliability by selecting a suitable transmission channel rate. Firstly, we design a statistical framework for single-link URLLC transmission where the channel information is incomplete and uncertain. The transmitter only has a limited data about the channel distribution. In this case, the statistical learning algorithm trains a PDF model based on the available data set and estimates the channel characteristics to guarantee the outage probability of URLLC packet transmission. Secondly, we estimate the cumulative distribution function (CDF) of channel signal-to-noise ratio (SNR) using KDE method and compare the results with the empirical CDF in [8]. The KDE is able to more accurately characterize the channel distribution with a small quantity of training samples. Finally, we compare the performance of different kernel density functions under variable kernel bandwidths. Then, we apply the Normal Kernel function to estimate the CDF of channel SNR and evaluate the mean outage probability and the confidence level in comparison with empirical estimator for different number of 
training samples. The results show that KDE can ensure that the target of URLLC outage probability is met with a higher probability even when limited data is available.

The rest of this paper is organized as follows. Section II describes the system model. Section III presents the principles of $\mathrm{KDE}$ and empirical $\mathrm{CDF}$ schemes. It also explains the statistical measurements of how the estimators maintain the target of URLLC outage probability. The simulation results are presented in Section IV. Finally, the conclusions are drawn in Section V.

Notations: We use $\mathbb{E}[$.$] and \mathbb{P}[$.$] to denote the expectation$ and the probability respectively. Also, $\operatorname{erf}($.$) represents the$ Gaussian error function.

\section{System Model}

We assume a downlink URLLC transmission system, where the transmitter (TX) sends URLLC packets at the selected transmission rate $R$ to the receiver (RX). Suppose $F$ represents the true CDF of the channel SNR denoted by the random variable $Y$. However, the TX has only limited knowledge of the channel distribution by collecting $n$ channel measurements prior to transmission (i.e. the number of training samples is denoted by $n$ ). The available training set is denoted by $\mathcal{X}^{n}=$ $\left\{x_{1}, \ldots, x_{n}\right\}$, where $x_{i}, \forall i=1, . ., n$, is univariate independent and identically distributed sample from the distribution $F$.

With a desired error probability $\xi$ at a given fixed finite blocklength $b_{l}$ to meet high reliability and low latency requirements, the URLLC maximal channel rate $r(Y)$ can be expressed as

$$
r(Y)=\log _{2}[1+Y]-\frac{1}{\ln 2} \sqrt{\frac{V}{b_{l}}} Q^{-1}(\xi),
$$

where $b_{l}$ represents the blocklength and $V$ denotes the socalled channel dispersion which satisfies $V=1-(1+Y)^{-2}$ [10]. The value of $V$ can be accurately approximated by one (i.e., $V=1$ ) when the threshold of error probability (i.e., $\xi$ ) is small or the value of blocklength $b_{l}$ is larger than 50 [11], [12]. $Q^{-1}(x)$ denotes the inverse of the Gaussian $Q$ function which is as $Q(x)=\frac{1}{\sqrt{2 \pi}} \int_{x}^{\infty} e^{\frac{t^{2}}{2}} d t$.

In the presence of channel uncertainty at the transmitter, a transmission rate could be selected that is larger than the URLLC channel rate $r(Y)$ and hence link outage would occur. To investigate the impact of channel uncertainty on the reliability of URLLC connection, we study the influence of link outage. The URLLC outage probability for transmission rate $R$ is defined as the probability that the channel rate $r$ is not sufficiently large to support the successful transmission rate $R$ as follows

$$
P_{\text {out }}(R)=\mathbb{P}[R>r(Y)]
$$

The aim is to maximize the transmission rate while guaranteeing a predefined reliability constraint such as

$$
P_{\text {out }}(R) \leq \epsilon,
$$

where $\epsilon$ denotes the URLLC target outage probability. In other words, the transmission rate $R$ is selected to ensure the URLLC outage probability constraint is satisfied. Therefore, when the true distribution $F$ is known, the maximum transmission rate of TX subject to (3) can be derived as

$$
R(\epsilon)=\log _{2}\left(1+F^{-1}(\epsilon)\right)-\frac{1}{\ln 2} \sqrt{\frac{V}{b_{l}}} Q^{-1}(\xi),
$$

where $F^{-1}(\epsilon)$ represents the $\epsilon$-quantile of $F$. Apparently, acquiring $R(\epsilon)$ depends on the availability of channel distribution knowledge. In the absence of perfect channel distribution knowledge, we propose a statistical learning approach based on kernel density estimation and the available training set $\mathcal{X}^{n}$.

\section{Non-PARAmeter Channel RATE SElECtion}

Parametric estimation is used for the case that the estimator knows the specified function of the operation system. For instance, when the channel distribution is perfectly known by the TX and the learning approach only needs to find the values of different parameters. In contrast, the practical wireless communication channel information is uncertain. Non-parametric estimation works on analyzing the case that the structure of the system is uncertain, and the learning data is statistical [13]. Non-parametric approach is independent of the specification bias and provides high flexibility when modeling a given data set, however, many of the non-parametric estimations are strongly influenced by the quantity of training data set.

\section{A. Kernel Density Estimation (KDE)}

$\mathrm{KDE}$ is a popular non-parametric estimation approach which is designed to improve the non-smooth PDFs under histogram estimation. As opposed to histogram estimation, KDE is independent of the width of bins and the start position of the bins by centring the kernel function at each data point. KDE can estimate the PDF of a random variable given a finite set of samples drawn from the distribution of interest [14].

Suppose the set of samples is $\mathcal{X}^{n}=\left\{x_{1}, \ldots, x_{n}\right\}$ which are drawn from an unknown PDF $f$. Applying KDE, the PDF of channel distribution given $\mathcal{X}^{n}$ can be estimated as

$$
\widehat{f}_{\mathrm{k}}(x)=\frac{1}{n h} \sum_{i=1}^{n} K\left(\frac{x-x_{i}}{h}\right),
$$

where $\widehat{f}_{\mathrm{k}}(x)$ represents the estimated PDF using KDE, $K($. is the kernel function, and $h>0$ is the smoothing bandwidth of KDE. The choice of bandwidth strongly influences the performance of kernel function [15], [16]. There are multiple choices for the kernel function, such as normal, epanechnikov, box and triangle functions. In this paper, we choose normal kernel function for the channel estimation since it is the most commonly used function and the performance of this function is the best in most cases. Assuming the mean value of $\mu=0$ and the standard deviation $\sigma$ for the Normal Kernel function, the estimated PDF in (5) can be rewritten as

$$
\widehat{f}_{\mathrm{k}}(x)=\frac{1}{n h \sigma \sqrt{2 \pi}} \sum_{i=1}^{n} e^{\frac{-\left(x-x_{i}\right)^{2}}{2 \sigma^{2}}}, \forall x_{i} \in \mathcal{X}^{n} .
$$


Since the training samples and SNR are non-negative, the estimated $\mathrm{CDF}$ using $\mathrm{KDE}$ (i.e., $\widehat{F}_{k}(y)$ ) can be expressed as

$$
\widehat{F}_{\mathrm{k}}(y)=\int_{0}^{y} \widehat{f}_{\mathrm{k}}(x) d x=\int_{0}^{y} \frac{1}{n h \sigma \sqrt{2 \pi}} \sum_{i=1}^{n} e^{\frac{-\left(x-x_{i}\right)^{2}}{2 \sigma^{2}}} d x .
$$

Let $u=\frac{x-x_{i}}{\sqrt{2} \sigma}$, thus, $\frac{d u}{d x}=\frac{1}{\sqrt{2} \sigma}, d x=\sqrt{2} \sigma d u$. Therefore, the estimated $\mathrm{CDF}$ using normal $\mathrm{KDE}$ can be derived as

$$
\widehat{F}_{\mathrm{k}}(y)=\frac{1}{2 n h} \sum_{i=1}^{n} \int_{0}^{y} \operatorname{erf}\left(\frac{x-x_{i}}{\sqrt{2} \sigma}\right) d x,
$$

where $\operatorname{erf}(x)=\frac{1}{\sqrt{\pi}} \int_{0}^{x} e^{-t} d t$ represents the Gaussian error function. Subsequently, based on the definition in (2), the expression of the outage probability can be written as

$$
\begin{aligned}
\widehat{P}_{\text {out }}\left(R \mid \mathcal{X}^{n}\right) & =\mathbb{P}\left[Y<2^{R+R F\left(b_{l}, \xi\right)}-1 \mid \mathcal{X}^{n}\right] \\
& =\widehat{F}_{\mathrm{k}}\left(2^{R+R F\left(b_{l}, \epsilon\right)}-1\right) .
\end{aligned}
$$

where $R F\left(b_{l}, \xi\right)$ represents the reliability factor function which is related to the URLLC transmission error rate and channel blocklength,

$$
R F\left(b_{l}, \xi\right)=\frac{1}{\ln 2} \sqrt{\frac{V}{b_{l}}} Q^{-1}(\xi) .
$$

Maintaining the reliability constraint on the outage probability (3), given the training set $\mathcal{X}^{n}$, the non-parametric KDEbased transmission rate selection can be derived as

$$
R_{\mathrm{k}}\left(\epsilon \mid \mathcal{X}^{n}\right)=\log _{2}\left(1+\widehat{F}_{\mathrm{k}}^{-1}(\epsilon)\right)-R F\left(b_{l}, \xi\right) .
$$

where $\widehat{F}_{\mathrm{k}}(\epsilon)$ denotes the estimated CDF of channel SNR using $\mathrm{KDE}$ when the outage target is $\epsilon$ and the training set is $\mathcal{X}^{n}$.

\section{B. Empirical CDF Estimation}

In statistics, the empirical CDF estimation is the distribution function associated with the empirical measure of a set of samples. Based on the empirical CDF estimation [8], assuming $Y$ is a non-negative random variable and the samples are drawn from an unknown distribution with $\mathrm{CDF} F$, the empirical CDF estimate can be written as

$$
\widehat{F}_{\mathrm{p}}(y)=\frac{1}{n} \sum_{i=1}^{n} 1_{x_{i} \leq y}, \forall x_{i} \in \mathcal{X}^{n}
$$

where $1_{x_{i} \leq y}$ represents a binary indicator function which is one if $x_{i} \leq y$. Consequently, (12) can be computed as

$$
\widehat{F}_{\mathrm{p}}(y)=\frac{i-1}{n}, x_{i-1} \leq y \leq x_{i}, \forall i=1, \ldots, n .
$$

Using the same rate-selection method as $\mathrm{KDE}$, given the training set $\mathcal{X}^{n}$, the estimated transmission rate based on empirical CDF estimation (denoted by $R_{\mathrm{p}}$ ) becomes

$$
R_{\mathrm{p}}\left(\epsilon \mid \mathcal{X}^{n}\right)=\log _{2}\left(1+\widehat{F}_{\mathrm{p}}^{-1}(\epsilon)\right)-R F\left(b_{l}, \xi\right)
$$

\section{Confidence Level of URLLC Outage Probability}

With these two kinds of estimators, the system would estimate appropriate transmission rates to make sure that URLLC outage probability is no larger than $\epsilon$. However, since we are using statistical estimation to control the transmission rate, the confidence level in ensuring the outage probability constraint is a variable depending on the size of the training set. We consider two measures similar to [8] to evaluate the achievable reliability with channel distribution uncertainty. The first one is the mean of estimated outage probability defined as

$$
\bar{P}_{\text {out }}=\mathbb{E}\left[\widehat{P}_{\text {out }}\left(R\left(\epsilon \mid \mathcal{X}^{n}\right) \mid \mathcal{X}^{n}\right)\right],
$$

where the expectation is taken over the distribution of the training sample $\mathcal{X}^{n}$.

The other measure is related to the level of confidence in the achieved reliability based on the CDF estimation. Let us define $\alpha$ as the probability that the URLLC outage probability is less than the threshold $\epsilon$ when TX estimates the rate $R$ with the set of training samples $\mathcal{X}^{n}$. The probability is computed with respect to the joint distribution of the training sample $\mathcal{X}_{n}$.

$$
\alpha(\epsilon)=\mathbb{P}\left[\widehat{P}_{\text {out }}\left(R\left(\epsilon \mid \mathcal{X}^{n}\right) \mid \mathcal{X}^{n}\right)>\epsilon\right]
$$

In statistics, $(1-\alpha(\epsilon))$ is called the level confidence and represents how often we satisfy the outage probability constraint considering different training data set $\mathcal{X}^{n}$ when the threshold of the URLLC outage probability is $\epsilon$. In the next section, we compare the performance of the two estimators, empirical and $\mathrm{KDE}$, in terms of two measures introduced earlier. The results reveal the number of samples $(n)$ required to achieve a certain level of confidence for the reliability constraint.

\section{Simulation Results}

We modeling the transmission channel under different kinds of channel fading (i.e., Rayleigh flat fading with the average power of $\lambda=1$ and Rician fading model with the shape factor $k \in\{0.5,2\}$, the shape factor $k$ shows the ratio between the average power of secular and diffuse components [8]). We select normal kernel function and set the mean value of normal distribution as $\mu=0$ and the standard deviation $\sigma=1$.

\section{A. Cumulative Distribution Function Estimation}

First, we assume the channel under Rayleigh flat fading, in which the distribution of $Y \sim F_{\lambda}$ follows an exponential distribution with the average SNR of $\mathbb{E}[Y]=\lambda$. The CDF of the channel SNR is derived as

$$
F_{\lambda}(y)=1-e^{-\frac{y}{\lambda}}
$$

We set the value of $\lambda=1$, thus, the training data set $\mathcal{X}^{n}$ is generated randomly drawing samples from an exponential distribution with mean value of one. After that we compare the accuracy of the estimated CDF when applying different KDE functions with the empirical CDF based on the root-meansquare deviation (RMSD). RMSD intuitively shows the difference between the predicted and theoretic values. The RMSD 


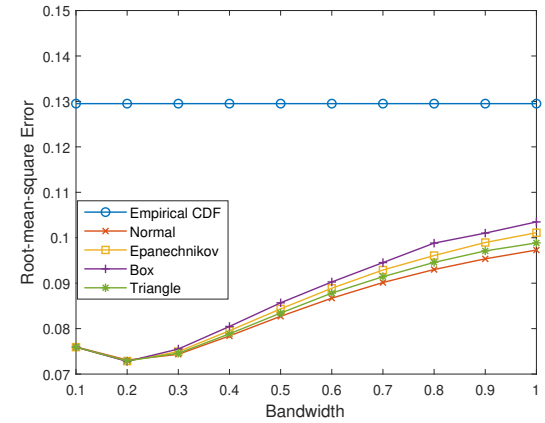

(a) $n=10$

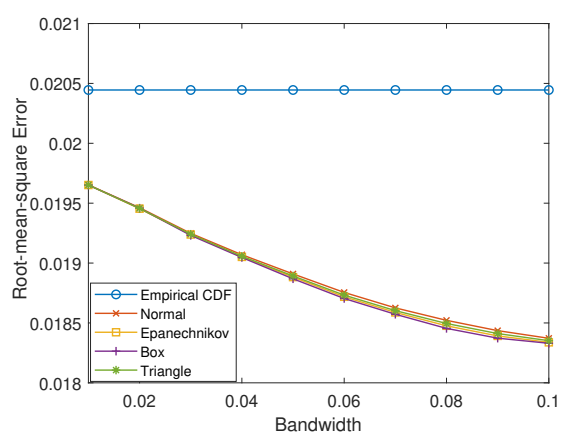

(b) $n=10^{2}$

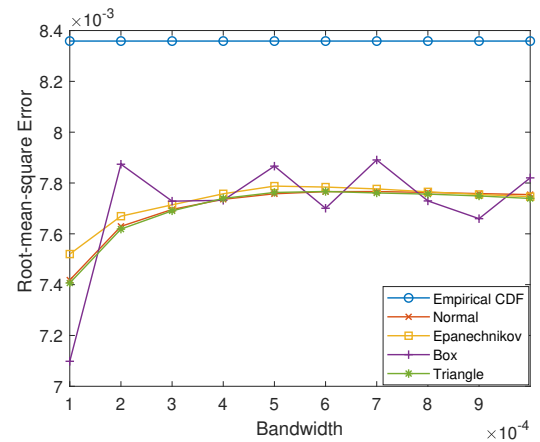

(c) $n=10^{3}$

Fig. 1. The root-mean-square errors of the estimated CDF versus kernel bandwidths under different kernel functions and training sample size ( $n$ )

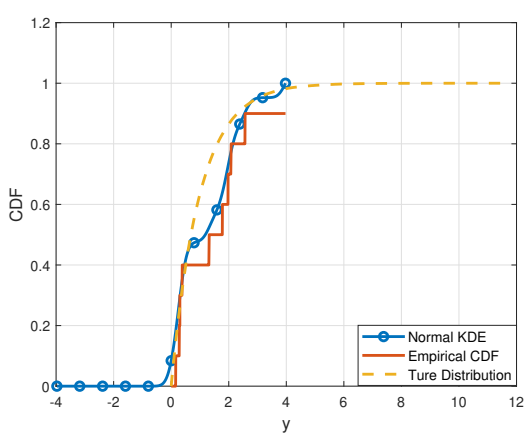

(a) $n=10$

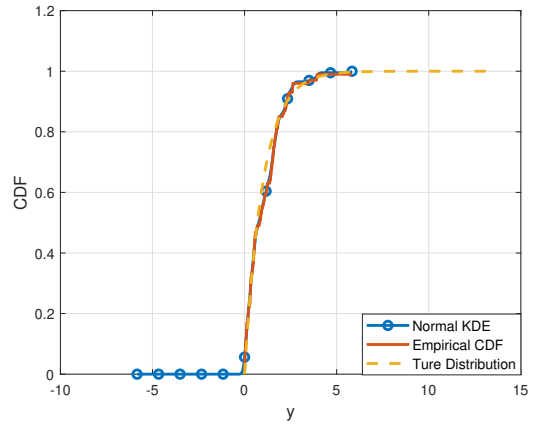

(b) $n=10^{2}$

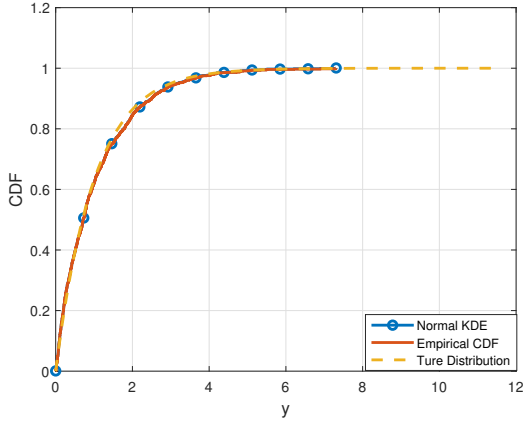

(c) $n=10^{3}$

Fig. 2. The estimated CDF by different kernel functions versus the training samples size $(n)$.

of empirical estimation $\left(R M S D_{\mathrm{p}}\right)$ and $\mathrm{KDE}\left(R M S D_{\mathrm{k}}\right)$ are calculated as follows

$$
\begin{aligned}
R M S D_{\mathrm{p}} & =\sqrt{\frac{1}{n} \sum_{i=1}^{n}\left(F\left(x_{i}\right)-\widehat{F}_{\mathrm{p}}\left(x_{i}\right)\right)^{2}} \\
& =\sqrt{\frac{1}{n} \sum_{i=1}^{n}\left(F\left(x_{i}\right)-\frac{i-1}{n}\right)^{2}}, \\
R M S D_{\mathrm{k}} & =\sqrt{\frac{1}{n} \sum_{i=1}^{n}\left(F\left(x_{i}\right)-\widehat{F}_{\mathrm{k}}\left(x_{i}\right)\right)^{2}} .
\end{aligned}
$$

Fig. 1 presents the values of $R M S D_{\mathrm{k}}$ when the system has various choice of kernel bandwidth $h$. Based on the results, when the channel has a limited number of training samples, KDE provides a more precise estimate of CDF with an appropriate kernel bandwidth selection. Besides, it is clear that $\mathrm{KDE}$ is less sensitive to the training sample size, which shows the stability and robustness of this method. Since the results using different Kernel density functions do not show a huge difference, in the following sections, we choose the normal kernel function for KDE channel estimation.

Fig. 2 shows $\mathrm{CDF}$ with an appropriate value of kernel bandwidth evident from Fig.1. From the results, CDF estimation using $\mathrm{KDE}$ is smoother and closer to the true distribution. However, when considering the shape of normal distribution, there is always a small probability for the negative values.
This problem can be solved by using a truncated probability density function. The truncated PDF can be derived as

$$
f^{\prime}(x)=\frac{g(x)}{1-F(x)}
$$

where,

$$
g(x)= \begin{cases}f(x), & x \geq 0 \\ 0, & x<0\end{cases}
$$

The truncated PDF can be used for large training set (e.g. when $n \geq 10^{3}$ ) as Fig. 2c, since the CDF for the negative values are quite small. However, for the small set of training data cases (i.e. $n \leq 10^{2}$ ), it is a better choice to keep the truth CDF.

\section{B. Non-parametric Outage Estimation}

Here, we investigate the average outage probability and confidence level in the reliability measure using KDE for different numbers of training samples $n \in\left\{10,10^{2}, 10^{3}, 10^{4}, 10^{5}\right\}$ and different values of $\epsilon \in\left\{10^{-3}, 10^{-4}\right\}$. The performance of empirical estimation is also investigated as a benchmark. To reduce the chance of re-transmission and maintain the two requirements of URLLC services, we set the value of transmission error rate is $\xi=10^{-9}$ and the quantity of channel blocklength is $b_{l}=200$. 


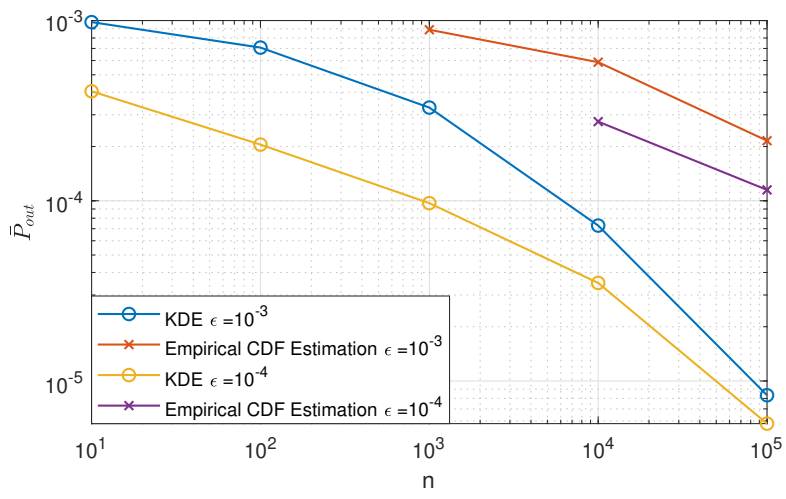

Fig. 3. Average outage probability versus training sample size $(n)$ under Rayleigh fading

In this part, we compare the learning performance under different channel fading models. The setting of Rayleigh fading model is the same as mentioned before. Considering the Rician $k$-factor as [8], the CDF of channel distribution is

$$
F_{\lambda, k}(y)=1-Q_{1}\left(\sqrt{2 k}, \sqrt{2 \frac{y}{\lambda}}\right)
$$

where $Q_{1}($,$) is the first order Marcum Q-function.$

To obtain $\bar{P}_{\text {out }}$, we have randomly generated $N$ training sets, each with a size of $n$. After obtaining the rate $\left(R\left(\epsilon \mid \mathcal{X}^{n}\right)\right)$ based on either (11) or (14) for a target $\epsilon$, we have generated $N$ independent realizations of $Y \sim F$. Then, for each SNR realization, we have compared the rate $R\left(\epsilon \mid \mathcal{X}^{n}\right)$ with $\log _{2}(1+$ $y)-R F\left(b_{l}, \xi\right)$ and repeated the comparisons for $N$ different training sets. Subsequently, $\bar{P}_{\text {out }}$ is computed as

$$
\bar{P}_{\text {out }}=\frac{1}{n N} \sum_{i=1}^{N} \sum_{j=1}^{n} 1_{\log _{2}\left(1+y_{i, j}\right)-R F\left(b_{l}, \xi\right)<R_{i}\left(\epsilon \mid \mathcal{X}_{i}^{n}\right)} .
$$

To compute $\alpha$ numerically, if the estimation of outage probability $\left(\bar{P}_{\text {out }}\left(R\left(\epsilon \mid \mathcal{X}^{n}\right) \mid \mathcal{X}^{n}\right)\right)$ for any training set excesses the target outage probability $\epsilon$, this will be counted in to find the $\alpha$ parameter. Therefore, the $\alpha(\epsilon)$ can be computed as

$$
\bar{\alpha}(\epsilon)=\frac{1}{N} \sum_{i=1}^{N} 1_{\widehat{P}_{\mathrm{out}, i}>\epsilon} .
$$

Firstly, we assume the channel under Rayleigh fading, Fig. 3 shows the mean values for URLLC outage probabilities $\left(\bar{P}_{\text {out }}\right)$ and Fig. 4 shows the confidence level $1-\bar{\alpha}(\epsilon)$ in URLLC outage probabilities. Comparing the results under the two kinds of estimators, it is clear with the empirical estimation a significantly larger number of training samples is required. From Fig. 3, it is evident that applying KDE for rate control will enable us to guarantee that the mean outage probability is lower that the target $(\epsilon)$ for a fairly small number of training samples. For instance, for $\epsilon=10^{-3}$, even with $n=10$ samples the mean outage is maintained at $10^{-3}$. Note for some cases there is no outage probability data for the empirical $\mathrm{CDF}$ estimation. This is because the selected transmission

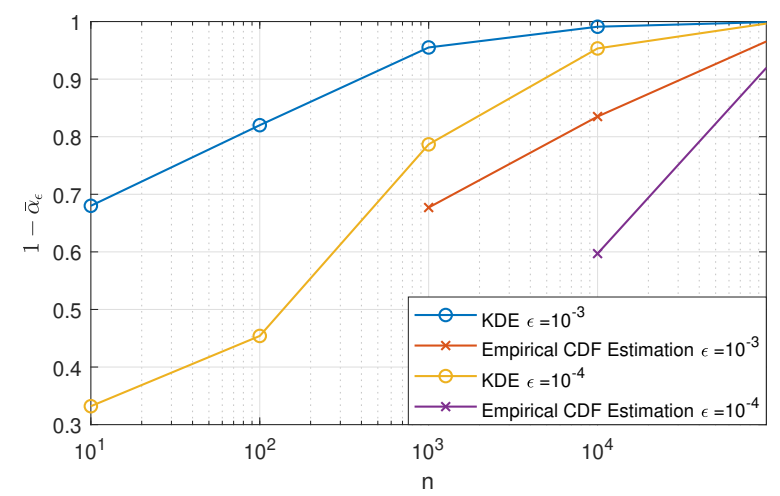

Fig. 4. Confidence level $(1-\bar{\alpha}(\epsilon))$ versus training sample size $(n)$ under Rayleigh fading

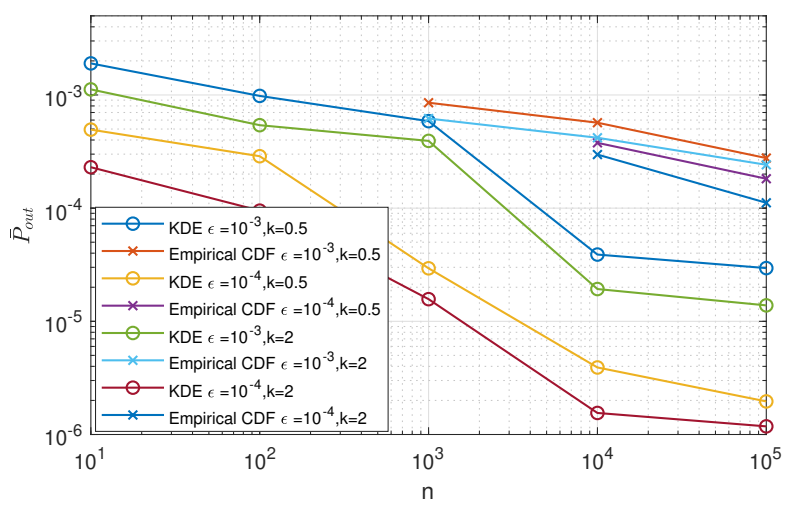

Fig. 5. Average outage probability versus training sample size $(n)$ under different Rician fading shape factors $(k)$.

rate is $R_{p}=0$ for those cases (i.e., when the number of training samples $n$ is smaller than $1 / \epsilon$ ). In Fig.4, it is shown that the confidence level is considerably higher using $\mathrm{KDE}$ approach. This empowers us to achieve ultra reliability even the transmitter only has a limited training data set. Fig. 5 and Fig. 6 shows the average outage probability and confidence level under Rician fading with different shape factors, both learning algorithms can have better estimation performance with a large value of shape factor $k$.

\section{Throughput Ratio}

To evaluate the rate selection performance, here we study the ratio between the average throughput given the training set and the true throughput with the assumption that the channel distribution is known. Therefore, we define this ratio as

$$
T(\epsilon, n)=\frac{\mathbb{E}\left[R\left(\epsilon \mid \mathcal{X}^{n}\right) 1_{R\left(\epsilon \mid \mathcal{X}^{n}\right) \leq \log 2(1+Y)-R F\left(b_{l}, \xi\right)}\right]}{R(\epsilon)(1-\epsilon)} .
$$

In (24), the throughput is defined as the average rate conditioned on that the channel is not in outage. Depending on how accurate the estimation is, $T(\epsilon, n)$ ranges between zero and one. Fig. 7 and Fig. 8 demonstrate $T(\epsilon, n)$ versus the training sample size under variable channel settings and 


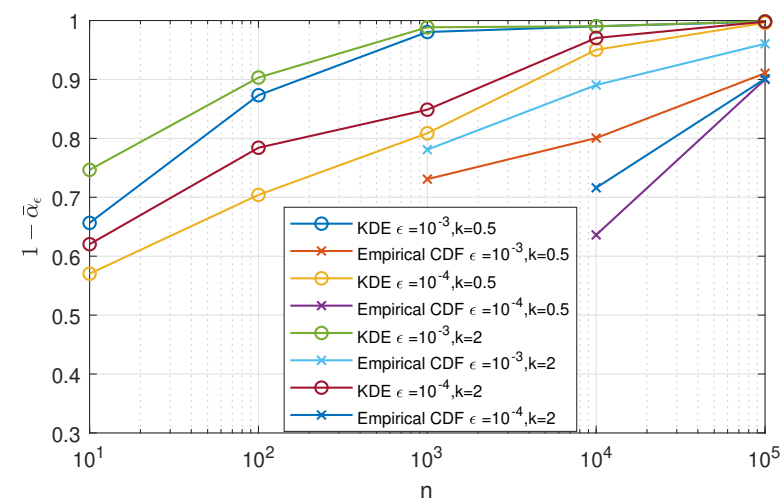

Fig. 6. Confidence level $(1-\bar{\alpha}(\epsilon))$ versus training sample size $(n)$ under Rician fading.

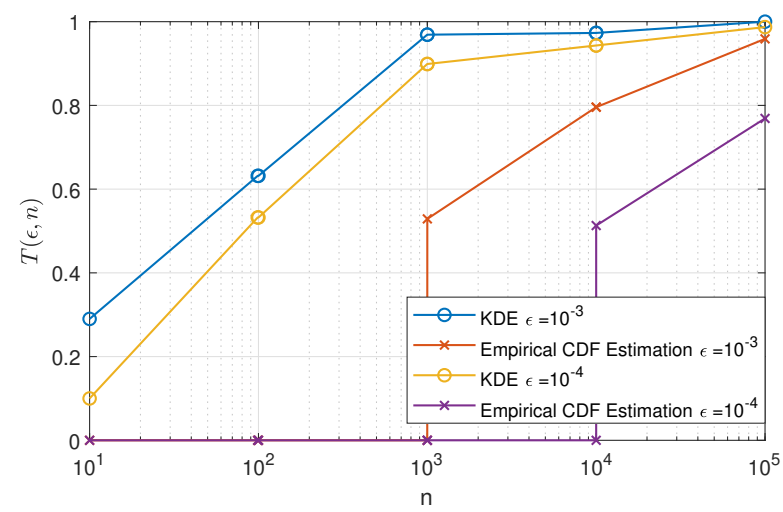

Fig. 7. Throughput ratio versus the training sample size under Rayleigh fading, $\xi=10^{-9}, b_{l}=200$.

different channel fading models. Evidently, $T(\epsilon, n)$ approaches to one much faster using $\mathrm{KDE}$ as compared with empirical estimation. In fact, with a small number of samples, KDE estimation achieves a relatively high throughput ratio. It is clear that the empirical estimation needs a minimum number of samples to support a non-zero throughput ratio, whereas for $\operatorname{KDE} T(\epsilon, n)$ is non-zero even with a small training sample and rapidly approaches one as $n$ increases. Additionally, a suitable choice of transmission parameters will improve the throughput ratio.

\section{CONCLUSION}

This work shows that the URLLC reliability is influenced by the availability and completeness of channel state information. In the presence of channel uncertainty, the system cannot ensure the outage probability is always smaller than a threshold. Therefore, to more accurately estimate the channel distribution, we propose KDE approach to estimate the CDF of channel SNR. Based on the results, the estimated CDF using KDE is more reliable than the empirical CDF estimation. The results show that the KDE can achieve a low outage requirement with high confidence even with a limited number of training samples as compared to the empirical method. Additionally, KDE is less sensitive to the number of training samples which reinforces its robustness.

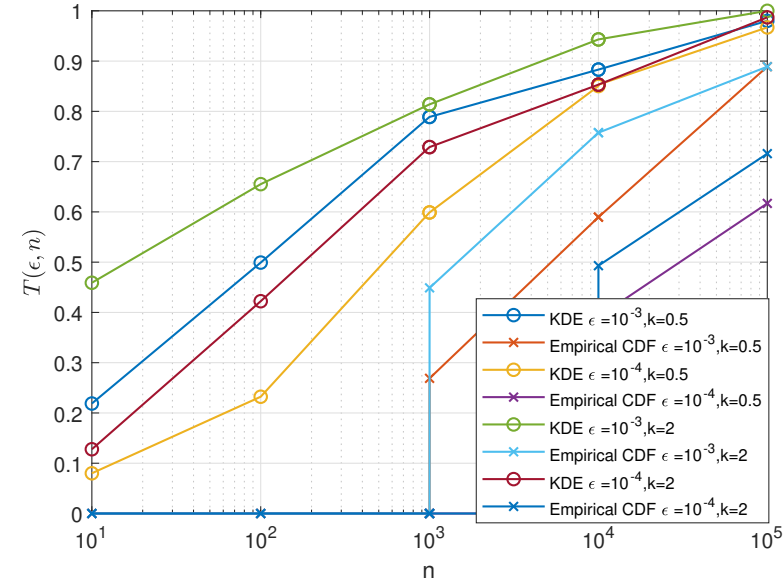

Fig. 8. Throughput ratio versus the training sample size under different Rician fading shape factors $(k), \xi=10^{-9}, b_{l}=200$.

\section{REFERENCES}

[1] P. Popovski et al, "Wireless access for ultra-reliable low-latency communication: Principles and building blocks," IEEE Network, vol. 32, no. 2, pp. 16-23, 2018.

[2] M. Bennis, M. Debbah, and H. V. Poor, "Ultra-reliable and low-latency wireless communication: Tail, risk, and scale," Proceedings of the IEEE, vol. 106, no. 10, pp. 1834-1853, Oct. 2018

[3] P. Popovski et al, "Wireless access in ultra-reliable low-latency communication (URLLC)," IEEE Transactions on Communications, vol. 67, no. 8, pp. 5783-5801, Aug 2019.

[4] "3GPP TR 38.913: Study on scenarios and requirements for next generation access technologies,," Mar. 2017.

[5] H. Ren, C. Pan, Y. Deng, M. Elkashlan, and A. Nallanathan, "Joint power and blocklength optimization for URLLC in a factory automation scenario," IEEE Transactions on Wireless Communications, vol. 19, no. 3, pp. 1786-1801, 2020.

[6] W. Zhang, M. Derakhshani, and S. Lambotharan, "Stochastic optimization of URLLC-eMBB joint scheduling with queuing mechanism," IEEE Wireless Communications Letters, pp. 1-1, 2020.

[7] P. C. F. Eggers, M. Angjelichinoski, and P. Popovski, "Wireless channel modeling perspectives for ultra-reliable communications," IEEE Transactions on Communications, vol. 18, no. 4, pp. 2229-2243, April 2019.

[8] M. Angjelichinoski, K. F. Trillingsgaard, and P. Popovski, "A statistical learning approach to ultra-reliable low latency communication," IEEE Trans. Commun., vol. 67, no. 7, p. 5153-5166, Jul. 2019.

[9] N. Mehrnia and S. Coleri, "Wireless channel modeling based on extreme value theory for ultra-reliable communications," arXiv preprint arXiv:2002.00565., 2002.

[10] Y. Polyanskiy, H. V. Poor, and S. Verdu, "Channel coding rate in the finite blocklength regime," IEEE Transactions on Information Theory, vol. 56, no. 5, pp. 2307-2359, 2010.

[11] L. Feng, Y. Zi, W. Li, F. Zhou, P. Yu, and M. Kadoch, "Dynamic resource allocation with RAN slicing and scheduling for uRLLC and eMBB hybrid services," IEEE Access, vol. 8, pp. 34 538-34 551, 2020.

[12] G. Durisi, T. Koch, and P. Popovski, "Toward massive, ultra-reliable, and low-latency wireless communication with short packets," Proceedings of the IEEE, vol. 104, no. 9, pp. 1711-1726, 2016.

[13] G. D. Durgin, T. S. Rappaport, and D. A. de Wolf, "New analytical models and probability density functions for fading in wireless communications," IEEE Transactions on Communications, vol. 50, no. 6, pp. 1005-1015, June 2002.

[14] G. R. Terrell and D. W. Scott, "Variable kernel density estimation," in The Annals of Statistics, 1992.

[15] S. J. Sheather and M. C. Jones, "A reliable data-based bandwidth selection method for kernel density," Journal of the Royal Statistical Society. Series B (Methodological), vol. 53, no. 3, pp. 683-690, 1991.

[16] S. T. Chiu, "Bandwidth selection for kernel density estimation," in The Annals of Statistics, 1991. 